\title{
Presencia de las bases de datos del CSIC en las bibliotecas universitarias y científicas españolas: descripciones y materiales didácticos para la formación de usuarios
}

Luis Rodríguez-Yunta*, María Jesús Sánchez-González *, José Ignacio Vidal-Liy*

CSIC databases in Spanish scientific and university libraries: learning materials and description for information literacy

\section{Las bases de datos del CSIC, un recurso con una importante presencia en los programas de formación de usuarios y alfabetización informacional}

Con la transformación de las bibliotecas académicas en Centros de Recursos para el Aprendizaje y la Investigación, las competencias formativas han cobrado un auge importante. Las bases de datos del CSIC constituyen uno de los productos que tradicionalmente han estado presentes en las selecciones de recursos de información utilizadas en las bibliotecas universitarias. Es lógico, por tanto, que mantengan esta presencia en los programas formativos y en los materiales para la autoformación que se ofrecen en estos centros. En esta contribución se muestra el resultado de la exploración realizada en junio de 2009. Se destaca por un lado, las diferencias encontradas en la orientación que se ofrece sobre estos recursos en los catálogos, las guías generales y las páginas de información de bibliotecas virtuales. Por último se analizan los principales materiales formativos localizados que se enfocan directamente a mejorar el manejo de las bases de datos del CSIC por los usuarios. La valoración de estos materiales facilita que sean utilizados como apoyo para las actividades formativas en otros centros.

* Unidad de Análisis Documental y Producción de Bases de Datos ISOC. Centro de Ciencias Humanas y Sociales, CCHS-CSIC. Correo-e: luis.ryunta@cchs.csic.es; mariajesus.sanchez@cchs.csic.es; nacho.vidal@cchs.csic.es

Recibido: 15-7-09. 
Con la denominación de bases de datos del CSIC hacemos referencia a tres productos específicos: ICYT (bibliografía española en Ciencia y Tecnología), ISOC (Humanidades y Ciencias Sociales) e IME (Biomedicina). Son recursos que se distribuyen en línea desde 1985, pero que a menudo han sido más conocidos en las bibliotecas a través de su edición en cd-rom por la empresa Micronet. Este producto, realizado actualmente en dvd, incluye bajo esta denominación común otros recursos: los catálogos colectivos de la red de bibliotecas (CIRBIC) y la base DATRI, producida por la CICYT, que recoge información sobre centros de investigación españoles, proyectos, patentes y resultados de investigación (aunque se trata de un producto que no está actualizado).

Otro motivo que puede causar cierta confusión radica en las diferencias en el producto debidas a la política de distribución. Existen dos plataformas de acceso a través de la web:

- Acceso gratuito: http://bddoc.csic.es:8080/. Permite consultar las bases de datos ICYT, ISOC e IME en versión sumarios, así como los directorios de revistas de las correspondientes disciplinas. También incluye la consulta en versión completa de la base de datos ISOC-Biblioteconomía y Documentación.

- Acceso por suscripción: http://bddoc.csic.es:8085/. Corresponde a la versión completa de todas las bases (ISOC, ICYT, IME) y directorios de revistas españolas.

El CINDOC ha sido el productor de ICYT e ISOC entre 1992 y 2007, asumiendo también su distribución junto con el IME, base producida por el Instituto de Historia de la Ciencia y Documentación López Piñero (instituto mixto Universidad de Valencia-CSIC). El CINDOC se creó por la anexión del instituto ISOC en el ICYT, en 1992. En 2007, un nuevo instituto, el IEDCYT, asumió sus principales líneas de investigación, mientras que la producción y distribución de bases de datos pasó a integrarse directamente en el Centro de Ciencias Humanas y Sociales, que agrupa a todos los institutos y servicios de estas disciplinas existentes hasta entonces dentro del CSIC en Madrid.

\section{Descripción de las bases de datos del CSIC en las páginas webs de las bibliotecas españolas}

Una exploración por las páginas web de las bibliotecas universitarias españolas muestra cuál es la visibilidad actual de las bases de datos del CSIC y la orientación que reciben los usuarios sobre su contenido y modo de acceso. A pesar del crecimiento de materiales didácticos de estas bases, tutoriales y, sobre todo, guías de uso, sin embargo se detectan algunos aspectos que no favorecen su difusión entre los estudiantes universitarios españoles. Sin ánimo de ser exhaustivos, sí se pretende a través de una serie de ejemplos mostrar un panorama, 
más o menos general, en lo relacionado con los problemas más comunes detectados en las mencionadas páginas web, y que dificultan la divulgación y uso de las bases de datos del CSIC.

De los problemas que se podrían listar, dos son los más extendidos o generalizados: el primero es la localización y acceso a las bases de datos del CSIC y el segundo la falta de actualización o incorrecciones en la información servida al respecto.

No es frecuente que las bases de datos, al menos las más relevantes, tengan un acceso rápido desde la misma homepage de la biblioteca. La de la universidad de Zaragoza es una excepción en este sentido y cuenta con uno de ellos a bases de datos que han considerado relevantes (Dialnet, Rebiun y sobre todo ISI), sin que consten en este apartado las bases del CSIC. Por lo general, el acceso a bases de datos bibliográficas suele encontrarse en una sección homónima de sus webs, aunque, en ocasiones, este mismo acceso puede formar parte de otras secciones, ya sea la de servicios de la biblioteca ya sea de la de recursos electrónicos. Sin embargo, la localización de las bases de datos del CSIC puede complicarse, no tanto por encontrar la sección concreta en la que se hallan las bases de datos, sino por la heterogeneidad en las formas de denominación utilizada. Así, hay bibliotecas que las tienen identificadas simplemente como bases del CSIC (Universidades de Cádiz y Santiago), otras que las tienen catalogadas sólo como ISOC, ICYT o IME y otras de las dos maneras y que disponen de un registro de entrada por cada una de las bases (Universidades de Barcelona, Autónoma de Barcelona, Valencia o Girona).

Si bien la localización y denominación de las bases del CSIC constituyen un problema importante a la hora de visibilizar, difundir y promocionar estas bases de datos, no lo es menos la información que se proporciona sobre las mismas. Por lo general en estos casos, la cuestión a tratar es doble: por una parte la información dada contiene incorrecciones y, por otra, parte es frecuente encontrar que los datos que se ofrecen no están actualizados.

Hay ocasiones en las que las bases de datos del CSIC quedan encasilladas como simples bases de sumarios similares a Dialnet o Compludoc (Universitat Rovira i Virgili), ocultando precisamente uno de sus valores añadidos más potentes, el análisis documental de contenido. La razón puede deberse a que la descripción se realiza a partir de la base de datos gratuita de sumarios. Es evidente en el caso de bibliotecas que no tienen suscripción a las bases del CSIC (Universidad de Cádiz), lo que conlleva que sus usuarios no reciban ninguna información sobre la existencia de un producto más completo.

No son pocas las universidades que en la descripción de las bases del CSIC, incluyen además de ICYT, ISOC e IME, las bases DATRI, CIRBIC-L y CIRBIC-R. Probablemente se arrastra esta información de un registro anterior referido al cd-rom, ya que estos productos no forman parte de la interfaz de consulta que se enlaza.

La información sobre las bases de datos bibliográficas producidas por el CSIC suele aparecer también en las guías realizadas por las bibliotecas universitarias en el apartado de formación de usuarios. Existen multitud de ejemplos de guías 
accesibles desde la página web de las bibliotecas y consorcios de bibliotecas universitarias que incluyen la descripción del contenido y estructura de las bases de datos del CSIC. Sin embargo, es frecuente que no se aclaren las modalidades de acceso a las bases que se oferta desde la biblioteca universitaria. No se distingue si el acceso se realiza desde el DVD o en línea. Este tipo de información puede ser muy útil para el usuario ya que el contenido y las actualizaciones difieren dependiendo de un modo u otro. También se echa de menos una clarificación sobre los dos tipos de acceso en línea (gratuito y por suscripción) y el detalle de las posibilidades que reporta para el usuario final.

Hay datos que podrían precisarse mejor en las descripciones de las bases de datos del CSIC existentes en los catálogos y guías generales de recursos de información mantenidas por las bibliotecas universitarias.

- Se dan cifras sobre número de registros que quedan obsoletas. Así, la universidad de Extremadura señala que ISOC tiene 300.000 registros, la mitad de los más de 600.000 actuales.

- Número de revistas especializadas que se analizan. En la actualidad los directorios de revistas recogen 2.453 en las áreas de Ciencias Sociales y Humanas, 775 en Ciencia y Tecnología, 494 en áreas de Biomedicina.

- La cobertura temporal, que se inicia en los años 70.

- La actualización, que es cuatrimestral para el DVD, pero diaria en el acceso en línea para ISOC e ICYT, e irregular en el caso del IME. En ocasiones existen confusiones en este aspecto, como la Universidad de La Laguna que indica en la parte de la información general que la actualización de las bases es cuatrimestral, para en la información particular de cada base, dar la información correcta, la actualización diaria.

- Tipología documental: fundamentalmente artículos de revistas científicas españolas y, en menor medida, actas de congresos y series de interés científico.

Se observa asimismo una escasa difusión de las funcionalidades que caracterizan a estos productos como herramientas útiles para los usuarios:

- Análisis documental del contenido, ya que en cada documento se realiza una indización y clasificación temática, lo que le otorga una potencialidad que no poseen las bases de sumarios como Dialnet o Compludoc.

- Sistema de selección y categorización de las revistas analizadas, en el caso de la base ISOC: http://bddoc.csic.es:8080/informacion. do?tabla=revi\&bd=ISOC.

- URL permanente para la cita de sus registros.

- Historial de búsquedas y amplias posibilidades para implementar estrategias complejas de recuperación. 
- Variedad de formatos de descarga: ficheros de texto, XML, RIS, RefWorks.

- Integración de enlaces SFX y OpenURL.

- Acceso a texto completo, mediante enlaces a la edición electrónica o petición de copia digitalizada (servicio de acceso al documento).

\section{Materiales formativos y de apoyo sobre el manejo de las bases de datos del CSIC, en línea}

El CINDOC, como productor y distribuidor, desarrolló unas páginas de ayuda que muchas bibliotecas universitarias enlazan como materiales de apoyo. Sin embargo, en otros casos, se ha optado por diseñar documentos propios dirigidos a la formación. Las guías de recursos y los tutoriales son parte de los servicios que pueden ofrecerse a los usuarios para que libremente puedan mejorar sus estrategias en el uso de los recursos para la búsqueda bibliográfica.

Se han localizado 17 materiales referidos específicamente a las bases de datos bibliográficas del CSIC, elaborados por 9 instituciones.

\section{Bases de dades del CSIC: Índice Médico Español (Presentació de la base de dades).}

Universitat Rovira i Virgili, Biblioteca d'Infermeria.

http://www.urv.cat/media/upload/arxius/urv/IME-tutorial-EUI.pps.

Completa presentación de la base IME en formato PowerPoint con una duración aproximada de 10 minutos. Desarrolla la explicación, tanto de la interfaz de acceso gratuito como de la de suscripción, comparando las opciones de ambas. Desarrolla ejemplos para la búsqueda y uso de todas las opciones básicas.

\section{Bases de datos bibliográficas del CSIC.}

Universidad de Málaga, Biblioteca Universitaria, abril de 2008.

Autor: Fernando Heredia Sánchez.

http://www.uma.es/publicadores/biblioteca/wwwuma/GuiaBDCSIConline.pdf.

Documento de 25 páginas en formato pdf. Es un magnífico tutorial, que hace un recorrido muy detallado por las características de las tres bases de datos, como entrar y como consultar. Explicita perfectamente la política de acceso y la diferencia entre las dos interfaces de usuario. Destaca de forma correcta la existencia de enlaces al texto completo. Desarrolla ejemplos de búsqueda, empleando las opciones disponibles. 


\section{FIGURA 1}

Página del tutorial «Bases de datos bibliográficas del CSIC», elaborado por la Universidad de Málaga que muestra en un ejemplo práctico de búsqueda, cómo limitar los resultados a documentos con acceso al texto completo (opción denominada filtro)

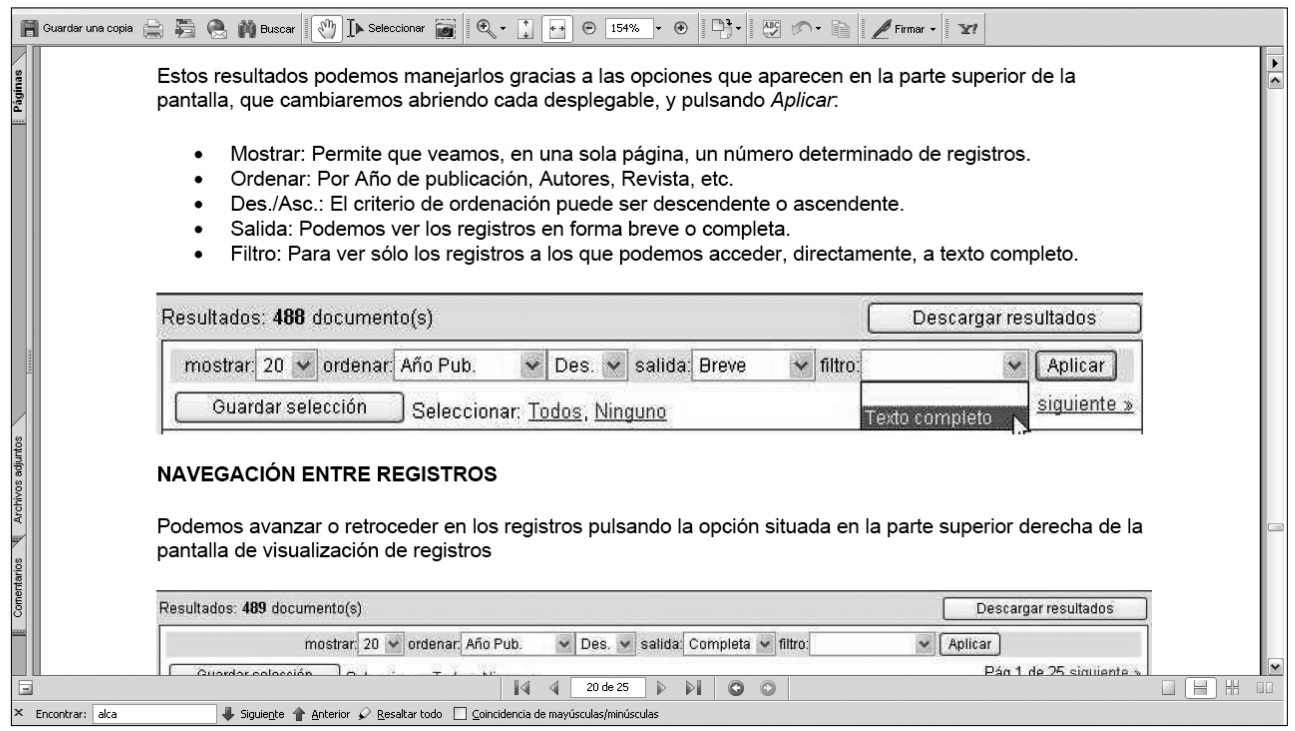

\section{Bases de datos Bibliográficas del CSIC. Guía de uso.}

Universidad de León, Biblioteca Universitaria, actualizada en octubre de 2008. http://biblioteca.unileon.es/guias/CSIC-Guia\%20de\%20uso.pdf.

Documento de 8 páginas en formato pdf. Es una guía básica sobre las opciones de búsqueda. No incluye ejemplos. Se indica la opción de filtro a documentos con disponibilidad de texto completo, pero no informa sobre el icono del enlace.

\section{Bases de datos del CSIC.}

Universidad de Murcia, Biblioteca Universitaria, mayo 2008.

Autoras: Rosario Guiard y Pepa Romero.

http://www.um.es/biblioteca/docs/TutorialCSIC.pdf.

Presentación en formato pdf que ofrece un repaso de todas las opciones de búsqueda, visualización, exportación. No explica que se puede acceder al texto completo, aunque se muestran ejemplos con el icono de enlace y se cita el menú mostrar sin decir que ofrece la opción de filtro.

\section{Las bases de datos del CSIC y el proceso para llegar al texto completo.} Universidad de Sevilla, Biblioteca de Ingenieros. http://bib.us.es/ingenieros/aprendizaje_investigacion/common/Flash/icyt.swf. 
Es un fichero en formato Adobe Flash. Se trata de un tutorial adaptado a la búsqueda en la base ICYT. Enseña a entrar desde la web de la biblioteca y desarrolla ejemplos de búsqueda. No indica que puede haber enlaces al texto completo, sino que orienta a los usuarios para localizar la publicación a través del catálogo de revistas de la universidad.

\section{Búsquedas en la Base de datos IME del CSIC.}

Universidad de Sevilla, Biblioteca de Centros de Salud, 2007.

http://bib.us.es/salud/aprendizaje_investigacion/common/guias_tutoriales/IME. swf.

Es un tutorial con algunos elementos interactivos, creado en formato Adobe Flash. Enseña a utilizar específicamente la base de datos IME, cómo acceder desde la biblioteca y realizar una estrategia de búsqueda. Explica de forma práctica la necesidad de utilizar sinónimos, el truncamiento y los operadores booleanos. Tras sacar los resultados en pdf, da a elegir entre dos opciones, la exportación a RefWorks o búsqueda de las revistas en el catálogo de la universidad. No hace ninguna mención a la existencia de enlaces al texto completo.

\section{FIGURA 2}

El tutorial interactivo "Búsquedas en la Base de datos IME del CSIC», realizado por la Universidad de Sevilla en formato flash

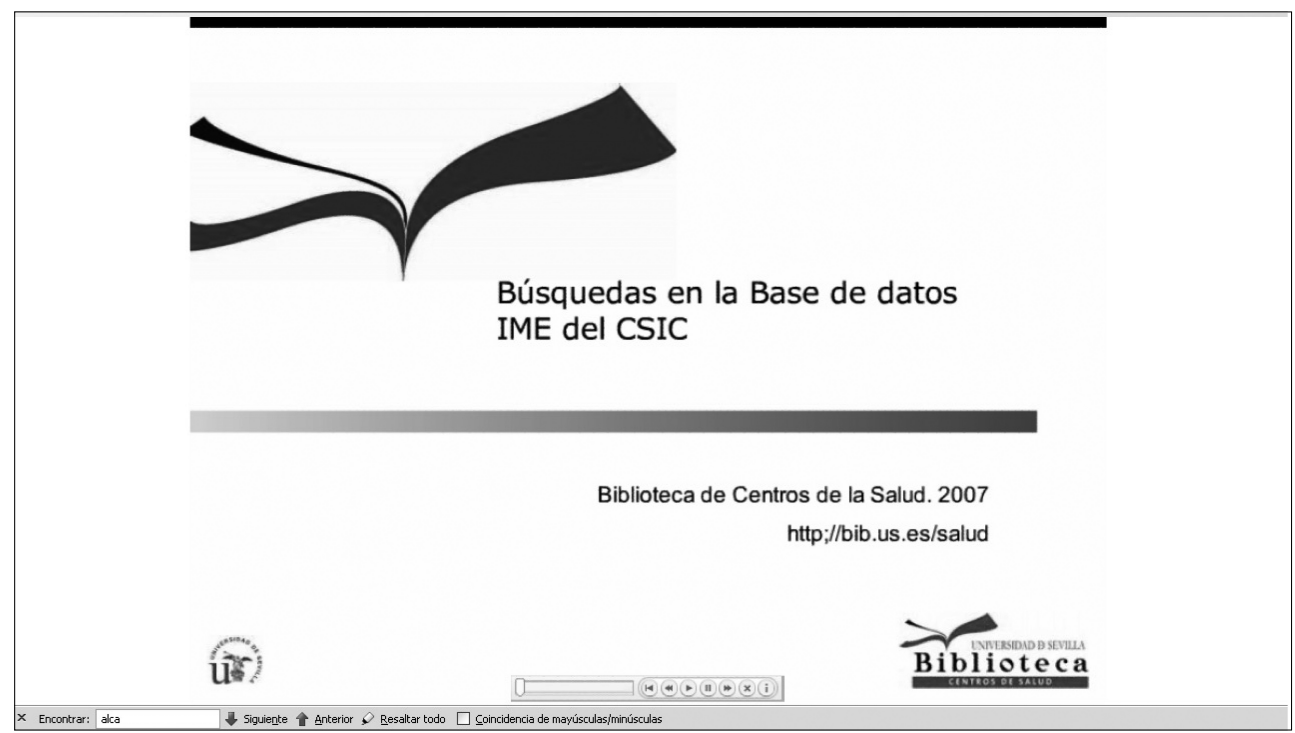

Cómo exportar registros desde IME en formato RIS.

Universidad de Sevilla, Biblioteca de Centros de Salud.

http://bib.us.es/salud/aprendizaje_investigacion/common/guias_tutoriales/exportar-desde-IME.swf. 
Es un fichero Adobe Flash (swf) que puede considerarse complementario del tutorial sobre Búsquedas en la Base de datos IME del CSIC. Indica exclusivamente como exportar los resultados de una búsqueda en formato RIS. Se trata de un material que ha quedado obsoleto, ya que indica el uso de este formato para la importación de las referencias en RefWorks, cuando en la actualidad la pasarela ya incluye una opción directa para este trasvase.

\section{Cómo utilizar el Historial en las bases de datos del CSIC.}

Universidad de Sevilla, Biblioteca de Centros de la Salud.

http://bib.us.es/salud/aprendizaje_investigacion/common/guias_tutoriales/Ime_ historial.swf

Es un fichero Adobe Flash (swf) que puede considerarse complementario del tutorial sobre Búsquedas en la Base de datos IME del CSIC. Indica exclusivamente como aplicar una estrategia a través de la combinación booleana en el historial de las búsquedas realizadas.

\section{Consulta de artículos de revistas en la base de datos CSIC (ISOC).}

Universidad de Sevilla, Biblioteca de Comunicación.

http://bib.us.es/comunicacion/aprendizaje_investigacion/common/Consulta_csic. swf.

Es un fichero en formato Adobe Flash que muestra un único ejemplo de búsqueda en ISOC. Explica cómo acceder desde esta biblioteca, pero ni siquiera desarrolla las opciones básicas que ofrece la interfaz.

\section{CSIC. Guía.}

UNED y Consorcio Madroño.

http://portal.uned.es/pls/portal/docs/PAGE/UNED_MAIN/BIBLIOTECA/ GUIAS\%20INVESTIGACION/GUIACSIC_0.PDF.

Guía elemental, documento de 2 páginas en pdf. No hace referencia a la opción de enlace al texto completo. Las nombra como bases de datos del IEDCYT.

\section{Exportación de registros bibliográficos desde las bases de datos del CSIC} a RefWorks.

Biblioteca de la Universidad Pablo de Olavide, Servicio de Información y Referencia.

http://www.upo.es/serv/bib/refworks/guias/refcsic.pdf.

Ayuda específica para la incorporación de referencias de las bases de datos del CSIC al gestor bibliográfico RefWorks. Es un documento de 2 páginas en formato pdf.

\section{Guia d'ajuda de ISOC- Ciències Socials i Humanitats.}

Universitat Rovira i Virgili, Biblioteca de Lletres, mayo de 2006.

http://www.urv.cat/media/upload/arxius/urv/guia_isoc.pdf.

Documento de 6 páginas en pdf que hace un recorrido por las opciones de la interfaz de búsqueda. Está en catalán. Es una guía básica que no añade conteni- 
dos a la ayuda disponible en la web de conexión. No hay ejemplos. No se dan explicaciones sobre la política de acceso. Se describe la interfaz limitada a suscriptores sin ninguna mención ni aclaración sobre los contenidos de la gratuita.

\section{Guia de base de dades del CSIC.}

Universitat Rovira i Virgili, Servei de Biblioteca i Documentació, 2006. http://www.urv.cat/media/upload/arxius/urv/guia_csic.pdf.

Documento de 3 páginas en pdf. Es una guía básica para orientar sobre su contenido y principales opciones. Se refiere al interfaz en línea, pero incluye por error la base de datos DATRI, que ya no figura entre las bases de datos del CSIC en este formato de acceso. No incluye ejemplos.

\section{Guía de consulta. Bases de datos del CSIC.}

Universidad de Burgos, Biblioteca Universitaria, Unidad de Información y Referencia Bibliográfica.

https://ubunet.ubu.es/webBD/descargaFichero.seu?fiche=csicguia.doc.

Guía básica. Tríptico a 3 columnas, documento en formato Word.

\section{Guía de uso de las Bases de Datos del Consejo Superior de Investigaciones Científicas.}

Universidad de Extremadura, Servicio de Documentación.

http://www.unex.es/teledoc/textosbd/impreso.pdf.

http://www.unex.es/teledoc/textosbd/guiacsicweb/index1.htm.

Es una guía básica de las opciones de búsqueda, pero ha quedado desfasada, pues no hace referencia al diseño actual. Tiene dos versiones, en pdf y html. La primera está firmada por Antonia García Arena, David Tejero Nogales y Luis Vicente Gordillo Tapia, y fechada en mayo de 2003.

\section{Guías de uso.}

Universidad Rey Juan Carlos.

http://www.urjc.es/z_files/ac_biblio/nuevaweb/bbdd/guias/i.htm.

Enlaza con guías específicas sobre ICYT, IME e ISOC. Son materiales obsoletos, que no hacen referencia al diseño actual de la interfaz de usuario.

\section{Guía rápida CSIC.}

Universidad de Las Palmas de Gran Canaria, Biblioteca Universitaria, abril 2008. http://biblioteca.ulpgc.es/files/repositorio_de_docum152/guias/recursos-e/Guia_ rapida_CSIC.pdf.

Documento en pdf de 5 páginas. Es una guía básica sobre las opciones de búsqueda. Se destaca la opción de exportación a RefWorks.

\section{ISOC - ICYT (CINDOC). Guía de consulta.}

Universidad Complutense de Madrid.

http://www.ucm.es/BUCM/cee/inforeco/InforEcoFlash/5/513.htm. 
Guía básica incluida dentro de InforEco, La Guía del Economista. Es un material obsoleto, ya que hace referencia a la interfaz propia de las bases ICYT e ISOC diseñada por la Universidad Complutense, que ya no está disponible.

\section{Tutorial Bases de Datos del CSIC.}

Universidad Pontificia de Salamanca. Biblioteca.

http://www.upsa.es/biblioteca/pdf/tutoriales/bbdd/tutorialcindoc.pdf.

Presentación de 13 páginas en formato powerpoint. Se dan pocos detalles sobre como aplicar una estrategia de búsqueda, pero sí hace referencia al acceso al texto completo y la exportación a RefWorks.

\section{Materiales formativos sobre el manejo de las bases de datos del CSIC, en cd-rom o dvd}

El uso del cd-rom (o su actual versión en dvd) ha perdido el papel predominante que tenía en la década de los noventa, cediendo el paso a la preferencia por el acceso en línea. Sin embargo, aún se han localizados cinco materiales que hacen referencia a esta versión.

\section{Bases de datos CSIC.}

Universidad de Málaga, Biblioteca de Informática y Telecomunicación.

Autora: Fuensanta López Pérez.

http://www.uma.es/publicadores/biblioteca/wwwuma/CdromCSIC.pdf.

Documento en pdf de cinco páginas. Es una guía básica sobre las opciones de búsqueda en el cd-rom.

\section{Bases de datos CSIC. Guía de uso.}

Universidad de Murcia.

http://www.um.es/biblio/Bases/guiacsic/index.html.

Páginas en HTML que muestran las opciones básicas del cd-rom. También figura alojada en la web de la Biblioteca de Ciencias de la Salud de Castilla-La Mancha: http://pagina.jccm.es/sanidad/ics/E.R.S.P/biblioteca/BD/manual_csic2004.pdf.

\section{Bases de datos del CSIC.}

Universidad Europea de Madrid, Biblioteca Dulce Chacón.

http://www.uem.es/biblioteca/web2006/coleccion_digital/guias_uso/csic.htm.

Página HTML que recoge las opciones básicas de uso del cd-rom.

\section{Bases de datos del CSIC. Guía básica de uso.}

Universidad Miguel Hernández de Elche.

Autores: Enrique Perdiguero y Carmen Sánchez Ardila (a partir de páginas de la Biblioteca de la Universidad de Murcia y de la Universidad de Valencia).

http://www.dsp.umh.es/docent/csic/index.htm.

Páginas en HTML que muestran las opciones básicas del cd-rom. 


\section{Guía de uso Base de datos del CSIC.}

Universidad de Navarra, Servicio de Bibliotecas.

http://www.unav.es/biblioteca/guias/csic.pdf.

Guía básica del cd-rom. Es un documento en pdf de siete páginas.

Nota: existen enlaces en la web a otras dos páginas ya desaparecidas, se trata de vínculos rotos a recursos de los que no hemos localizado una versión actual:

— Guía de uso del cd-rom: http://biblioteca.unirioja.es/biblio/bdg/bdgcsic.pdf.

— Manual CSIC: http://www.uv.es/ infobib/guias/manual_csic.html. 
